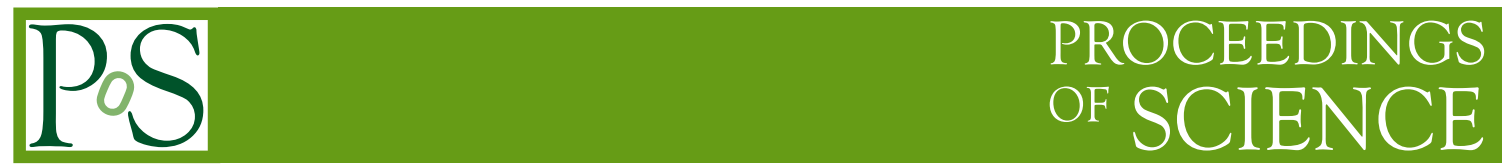

\title{
Updates of PDFs in the MMHT framework
}

\section{R.S. Thorne*}

Department of Physics and Astronomy, University College London, WC1E 6BT, UK

E-mail: robert.thorne@ucl.ac.uk

\section{S. Bailey}

Rudolf Peierls Centre, Beecroft Building,

Parks Road, Oxford, OX1 3PU, UK

E-mail: shaun.bailey@physics.ox.ac.uk

\section{T. Cridge}

Department of Physics and Astronomy,

University College London, WC1E 6BT, UK

E-mail: t.cridge@ucl.ac.uk

\section{L.A. Harland-Lang}

Rudolf Peierls Centre, Beecroft Building,

Parks Road, Oxford, OX1 3PU, UK

E-mail: lucian.harland-lang@physics.oxford.ac.uk

\section{A.D. Martin}

Institute for Particle Physics Phenomenology,

University of Durham, DH1 $3 L E, U K$

E-mail: A.D.Martinedurham.ac.uk

\section{R. Nathvani}

Department of Physics and Astronomy,

University College London, WC1E 6BT, UK

E-mail: ricky.nathvani.15ducl.ac.uk

\begin{abstract}
We summarise recent developments in the path towards the "MMHT19" parton distribution functions. We concentrate on the extraction of the strange quark upon the improvement of theoretical calculations for NNLO charged current cross sections; the effect of an extension of our parameterisation; and the role of correlated uncertainties in some data sets which prove difficult to fit.
\end{abstract}

XXVII International Workshop on Deep-Inelastic Scattering and Related Subjects - DIS2019

8-12 April, 2019

Torino, Italy

${ }^{*}$ Speaker. 
The MMHT2014 PDFs [1] were the last major update in the MRST/MSTW/MMHT family of PDFs. They included a variety of LHC data in their determination, but at that point the constraint on the PDFs was almost entirely from older fixed target and HERA DIS data, and some Drell-Yan and jet data from Tevatron experiments. Soon after the publication of the MMHT2014 PDFs we studied the effect of including the final HERA total cross section measurements [2], noting only minor changes in the central values and uncertainties [3]. The inclusion of a wide variety of new LHC data was considered in [4]. Most new data was fit well, and produced relatively small changes in both the central values and the uncertainties of the PDFs, most notably in the decomposition into flavours and into valence and sea quarks. A more significant change was induced when the ATLAS $W, Z$ data in [5] were included. A relatively good fit could be achieved $(\sim 110 / 61)$, but this required a modification of the small- $x$ valence quarks and, in particular, an increase in the strange quark. This latter change was less pronounced than that found by ATLAS in [5], probably due to the inclusion of fixed target charged current DIS dimuon production data [6], which is the traditional type of constraint on the strange quark in global PDF fits and prefers a lower value than the ATLAS data.

\begin{tabular}{|l|c|c|c|c|}
\hline & $\mathrm{BR}(c \rightarrow \mu)$ & $\mathrm{CCFR} / \mathrm{NuTeV} \chi^{2}$ & ATLAS $W, Z \chi^{2}$ & Total $\chi^{2}$ \\
\hline MMHT+HERAII & 0.090 & 120.5 & & 3526.3 \\
MMHT+HERAII (NNLO dimuon ) & 0.102 & 122.7 & & 3527.3 \\
MMHT+HERAII (NNLO VFNS dimuon) & 0.101 & 123.9 & & 3531.3 \\
MMHT+HERAII+ATLAS $(W, Z)$ & 0.073 & 127.3 & 108.6 & 3684.7 \\
MMHT+HERAII+ATLAS $(W, Z)$ (NNLO dimuon ) & 0.084 & 137.8 & 106.8 & 3688.4 \\
MMHT+HERAII+ATLAS $(W, Z)$ (NNLO VFNS dimuon) & 0.086 & 137.0 & 106.8 & 3688.5 \\
\hline$N_{p t s}$ & & 126 & 61 & 3337 \\
\hline
\end{tabular}

Table 1: The branching ratio for charm mesons to muons, and the $\chi^{2}$ values for fit variants before and after the inclusion of the data in [5] and the correction to the NNLO dimuon cross sections.

However, the full NNLO corrections to heavy flavour charged current DIS have only recently been calculated [7], and all PDF analyses so far have used either NLO cross sections, or NNLO approximations (as in MMHT), in NNLO PDF extractions. The NNLO corrections are of order $-10 \%$ in the region $x=0.01$ relevant for the main strange quark sensitivity of the ATLAS data. This implies a larger strange quark may be needed to best fit the dimuon data and better potential compatibility with the ATLAS data. We have included this full NNLO calculation in a complete PDF fit for the first time, and modified our variable flavour number scheme (VFNS) for charged currents to include the calculation, which is for fixed flavour number (this required some minor corrections to the details of our charged current VFNS). The results of the fit quality are shown in Table 1. We note that when the ATLAS $W, Z$ data are not included the inclusion of the correct NNLO makes little difference to fit quality, though it does raise the preferred value of the branching ratio for $c \rightarrow \mu$ in the dimuon fit a little, consistent with a smaller charm cross section. The input value of the branching ratio is $0.092 \pm 10 \%$, very consistent with these fits. When the ATLAS $W, Z$ data are added using the standard MMHT treatment of dimuons the fit to the dimuon data deteriorates a little, but the branching ratio also has to take a very low value of 0.073 to accommodate the raised strange quark. With the full NNLO corrections the dimuon fit actually worsens, but that to ATLAS $W, Z$ data improves slightly and the global $\chi^{2}$ changes by less than 5 units. The branching ratio increases back to 0.086 , however, demonstrating more genuine compatibility between the fit to dimuon data and to ATLAS data. The effect on $s+\bar{s}$ is shown in Fig. 1 . With both the old 
MMHT treatment and the corrected NNLO cross section inclusion of the ATLAS $W, Z$ data raises the strange quark for $x$ above 0.001 and reduces the uncertainty. However, the rise is a little less significant when the full NNLO is used, with the updated strange central value straying outside the one sigma error band of the fit without ATLAS $W, Z$ data only marginally near $x=0.02$.
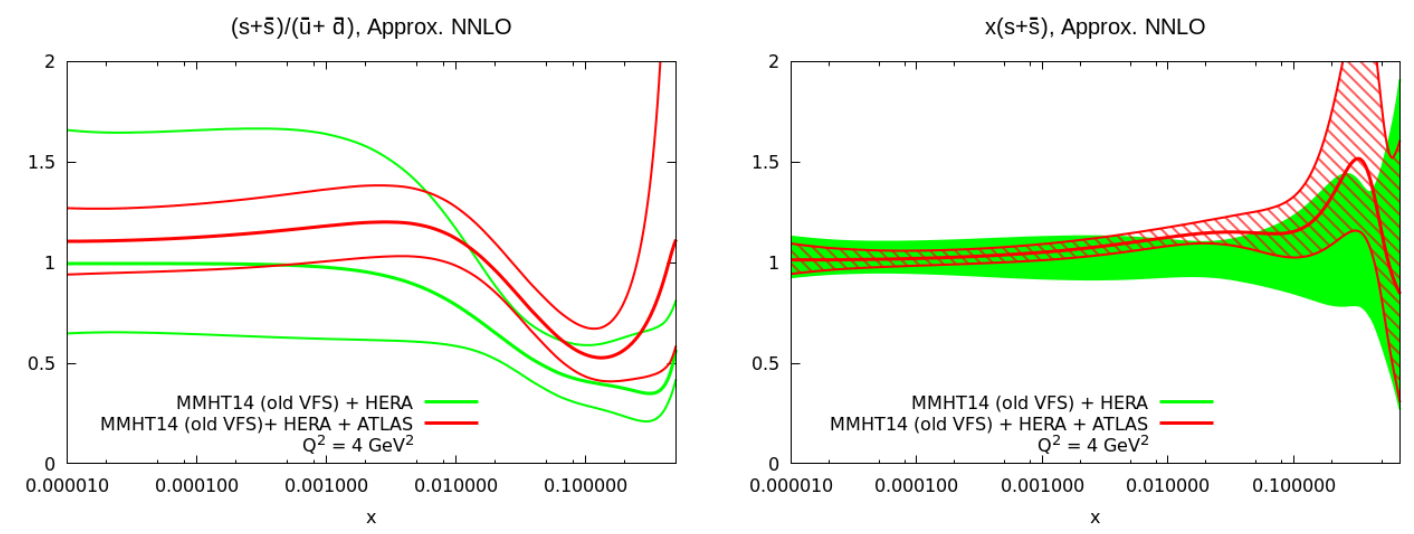

$(\mathrm{s}+\overline{\mathrm{s}}) /(\overline{\mathrm{u}}+\mathrm{d})$, Exact NNLO
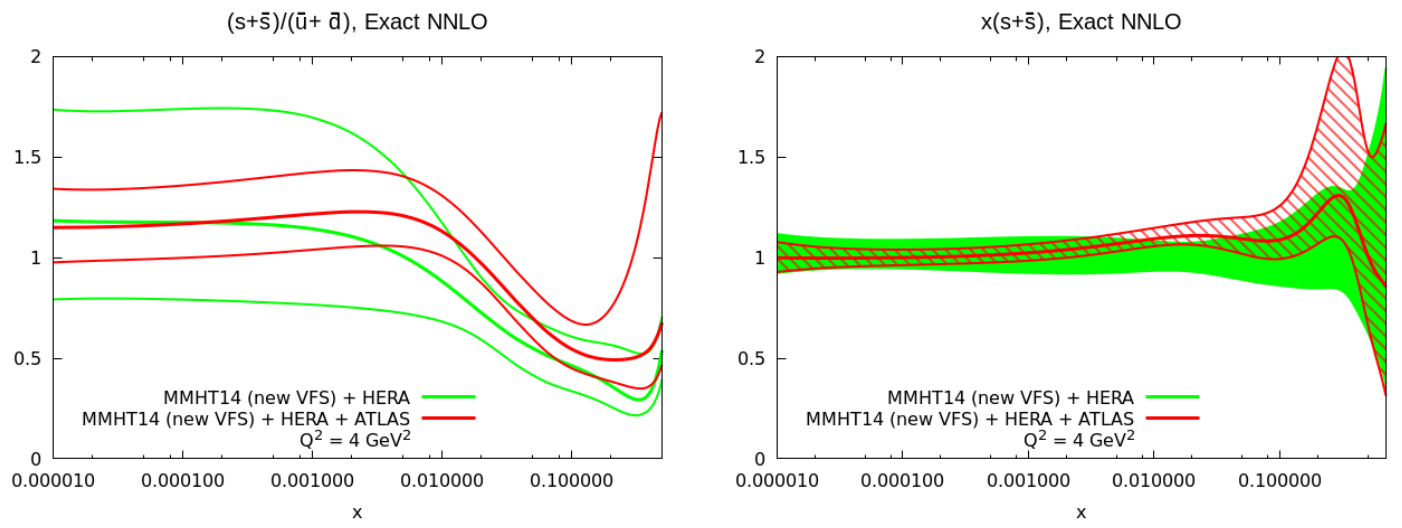

Figure 1: The strange over the light quark average and change in strange on addition of ATLAS $W, Z$ data without full NNLO as in MMHT2014 (top), and with full NNLO and the updated VFNS (bottom).

With the inclusion of more constraining LHC data we examine the introduction of more free parameters in our functions for the input PDFs. In [8] we changed our previous parameterisation to one with most PDFs parameterised as $A(1-x)^{\eta} x^{\delta}\left(1+\sum_{i=1}^{n} a_{i} T_{i}\left(1-2 x^{\frac{1}{2}}\right)\right)$, where $\left.T_{i}\left(1-2 x^{\frac{1}{2}}\right)\right)$ are Chebyshev polynomials. We also examined how many were needed to fit data of a given precision. At the time it was concluded that 4 (at most) was sufficient. However, for $(\bar{d}-\bar{u})\left(x, Q_{0}^{2}\right)$ by default we still used the old input form with only 4 parameters in total. We now try putting this distribution on an equal footing with the other PDFs by using Chebyshev polynomials, i.e.

$$
(\bar{d}-\bar{u})\left(x, Q_{0}^{2}\right)=A(1-x)^{\eta_{s e a}+2} x^{\delta}\left(1+\gamma x+\Delta x^{2}\right) \rightarrow A(1-x)^{\eta_{s e a}+2} x^{\delta}\left(1+\sum_{i=1}^{m} a_{i} T_{i}\left(1-2 x^{\frac{1}{2}}\right)\right),
$$

where we first choose $m=4$. This improves the global fit by about 10 units and eases tension between ATLAS $W, Z$ data and E866 Drell Yan ratio data [9].

However, from the studies in [8] it was clear that once PDF precision of greater than $1 \%$ was possible 5 or 6 polynomials would be required. Hence we extend the parameterisation for $u_{V}, d_{V}$, sea, $(\bar{d}-\bar{u})$ and $(s+\bar{s})$ sequentially to the form with $n=6$ (though for $(s+\bar{s})$ the small- $x$ power and two coefficients remain tied to the sea). The gluon parameterisation is of a different 
form, with two separate terms, but here we increase the free parameters from 7 to 9 . Compared to MMHT2014, this increases our number of free parameters in total from 36 to 50. The main improvements in the fit $\chi^{2}$ (to essentially the same data as in [4]) are after the extension to 6 polynomials of $(\bar{d}-\bar{u})\left(x, Q_{0}^{2}\right)$, from additionally extending $d_{V}\left(x, Q_{0}^{2}\right)\left(u_{V}\left(x, Q_{0}^{2}\right)\right.$ is not significant), and $g\left(x, Q_{0}^{2}\right)\left(\operatorname{sea}\left(x, Q_{0}^{2}\right)\right.$ and $s^{+}\left(x, Q_{0}^{2}\right)$ are not significant). The improvements to individual data sets are shown in Table 2. There is a reduction in tension between DY ratio data and LHC data, and an improvement in LHC lepton asymmetry data, and some fixed target deuteron data. The improvement due to the gluon is spread over data sets and due only partially to HERA data.

\begin{tabular}{|c|c|c|c|}
\hline Data set & $-\Delta \chi^{2} \quad(\bar{d}-\bar{u})$ & $-\Delta \chi^{2} \quad(\bar{d}-\bar{u}), d_{V}$ & $-\Delta \chi^{2}$ All \\
\hline Total & 17.6 & 34.0 & 48.9 \\
\hline $\mathrm{BCDMS} F_{2}^{p}$ & -4.6 & -3.3 & -2.7 \\
\hline $\mathrm{BCDMS} F_{2}^{d}$ & -2.7 & 4.9 & 8.5 \\
\hline $\mathrm{NMC} F_{2}^{n} / F_{2}^{p}$ & 6.5 & 6.1 & 6.0 \\
\hline 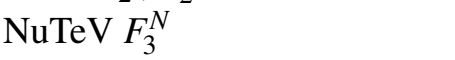 & -0.3 & 1.7 & 3.2 \\
\hline $\mathrm{E} 866 \sigma(p d) / \sigma(p p)$ & 8.2 & 10.1 & 11.0 \\
\hline NuTeV dimuon & 0.7 & 1.0 & 3.0 \\
\hline HERA I+II $\sigma\left(e^{+} p\right) 920 \mathrm{GeV}$ & 1.1 & 1.7 & 4.6 \\
\hline $\mathrm{CMS} p p \rightarrow l^{+} l^{-}$ & 0.7 & 1.8 & 3.1 \\
\hline $\mathrm{D} 0 \sigma\left(e^{+}\right)-\sigma\left(e^{-}\right)$ & -1.2 & -3.4 & -1.4 \\
\hline $\operatorname{CMS} 8 \mathrm{TeV} \sigma\left(l^{+}\right)-\sigma\left(l^{-}\right)$ & 4.4 & 5.0 & 4.6 \\
\hline ATLAS $7 \mathrm{TeV} W, Z$ & -0.5 & 2.2 & 4.3 \\
\hline CMS $7 \mathrm{TeV}$ jets & -0.5 & 0.2 & 3.2 \\
\hline
\end{tabular}

Table 2: The $\chi^{2}$ improvement for various data sets when extra parameters are added.

When determining the PDF uncertainties we go from 25 eigenvector pairs in MMHT2014 to 30 , one extra parameter for each PDF other than the light sea (and $s-\bar{s}$ ). 20 parameters are fixed at their best fit values - any extra eigenvectors would be highly non-quadratic and lead to very little extra uncertainty. The mean tolerance $T=3.31$, similar to MMHT2014. 27 eigenvector directions are constrained primarily by LHC data sets, largely 7 TeV ATLAS $W, Z$ data and CMS $W$ data. E866 Drell Yan asymmetry is vital for constraining $\bar{d}-\bar{u}$. Tevatron data of various types are the primary constraint for 8 eigenvectors, and fixed target DIS data still constrains 12 eigenvectors (mainly high- $x$ quarks). Hence, a fully global fit is necessary for full constraint. Some of the resulting PDFs are shown in Figs. 2 and 3. There is a significant change in the shape of the $d_{V}$ distribution. The uncertainty at both large and small $x$ increases due to the extra freedom. $d_{V}$ interplays with $(\bar{d}-\bar{u})$, also shown in Fig. 2, and the latter becomes flatter over the range $x=0.01-0.1$. The gluon, shown in Fig.3, stays well within previous uncertainties, except at very high $x$ where the uncertainty is huge and larger with the new parameterisation. $\bar{u}$, also shown in Fig. 3, is generally a little smaller, due to the increase in $(s+\bar{s})$ already discussed, and again has increased uncertainty at $x>0.6$. We note that despite more freedom in $(\bar{d}-\bar{u})$ the parameterisation still leads to a very quick approach to 0 at low $x$. This is likely unphysical, and we have recently tried parameterising $\bar{d} / \bar{u}$ instead, using the same number of parameters. Results are preliminary, but a small decrease in $\chi^{2}$ occurs, with a greater uncertainty as $x \rightarrow 0$ (though $\bar{d} / \bar{u} \rightarrow 1$ to very 
good accuracy), and with a ratio of $\bar{d} / \bar{u}<1$ at low $x$, but only at about one sigma level.

$d_{v}(N N L O)$ percentage change from MMHT14 at $Q^{2}=100 \mathrm{GeV}^{2}$

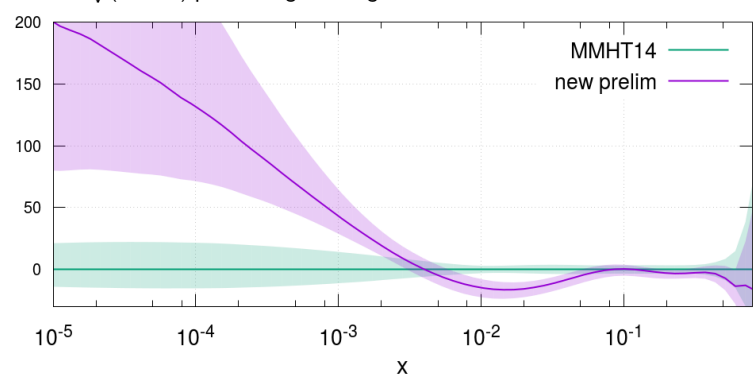

$\bar{d}-\bar{u}(N N L O)$ at $Q^{2}=100 \mathrm{GeV}^{2}$

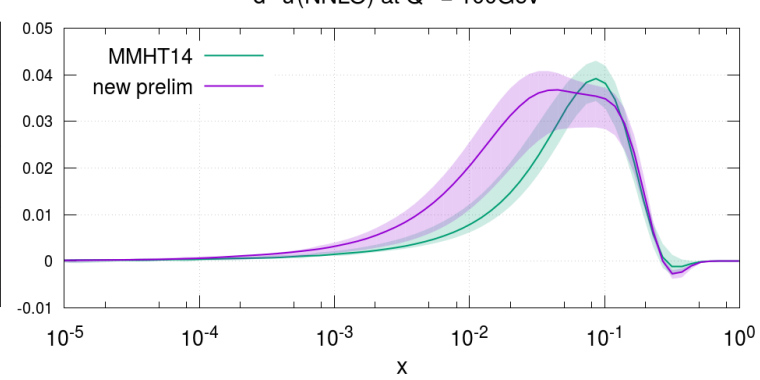

Figure 2: The NNLO down valence (left) and $\bar{d}-\bar{u}$ (right) for the fits with extended parameterisation.
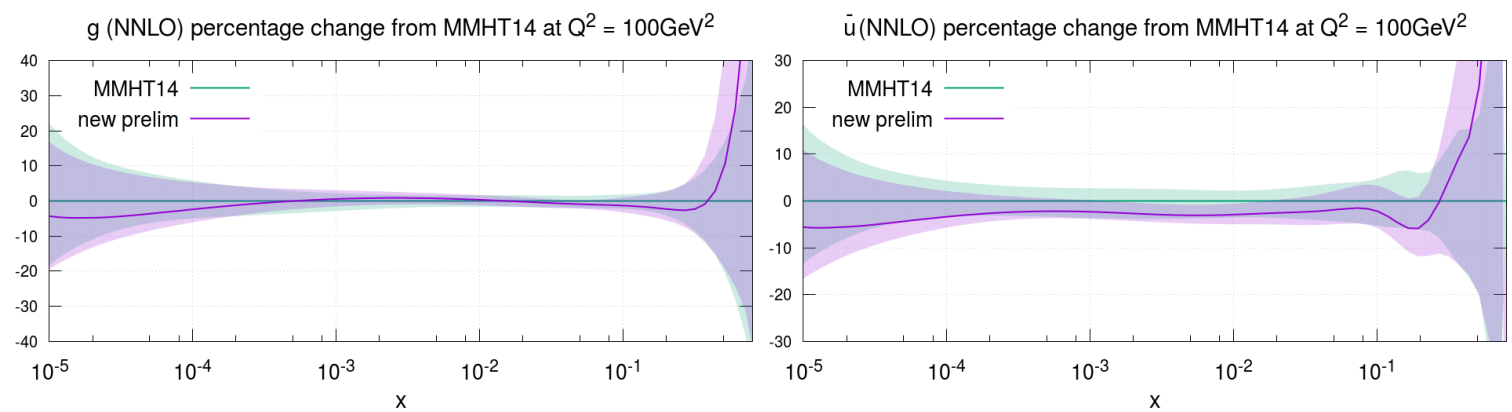

Figure 3: The NNLO gluon (left) and $\bar{u}$ (right) for the fits with extended parameterisation.

As well as these main results on the way to presenting the new updated "MMHT2019" PDFs, we note some other recent developments. In a previous study [10] we found difficulty in fitting ATLAS $7 \mathrm{TeV}$ inclusive jet data [11], even with full NNLO [12]. To alleviate this we investigated decorrelation of some systematic uncertainties between rapidity bins, finding dramatic improvement is we consider just two uncertainties, and little sensitivity of the gluon to the decorrelation procedure. This led to more sophisticated proposals for similar methods in [13]. We have also recently tried fitting to more than one of the top quark differential distributions in [14], which is possible due to the knowledge of statistical correlations between distributions[15], again using full NNLO [16]. We see a similar picture, i.e. one can fit some individual distributions well, but not simultaneously. This is due to inconsistencies in the required shifts related to correlated systematics (the same result is noted and studied in [15] and by the CT collaboration). In this case the important systematics are all related to Monte Carlo generation, and not obviously as well correlated as presented. Significant improvement can be achieved on relaxation of correlations. However, in this case the gluon is more sensitive to the details of the treatment, and some distributions require decorrelation within that particular distribution to enable a good fit. More details will be presented in the future.

We also note that we have completed the study of the inclusion of QED effects and the photon parton distribution [17]. We use essentially the PDFs of [3] as a baseline and use the LUX [18, 19] procedure for the input photon, making it fully consistent with the MMHT framework, including the production of the photon PDF in the neutron. We also examine the impact on high-mass DrellYan data, noting that the photon initiated contributions are sometimes smaller than the QED effects on quark evolution. We have made grids available, and in particular have separated the elastic and inelastic contributions to the photon PDF. 


\section{Acknowledgements}

LHL thanks the Science and Technology Facilities Council (STFC) for support via grant award ST/P004547/1. RST and TC thank the Science and Technology Facilities Council (STFC) for support via grant awards ST/P000274/1. RN thanks the Spreadbury Fund. SB thanks the STFC.

\section{References}

[1] L. A. Harland-Lang, A. D. Martin, P. Motylinski and R. S. Thorne, Eur. Phys. J. C 75 (2015) no.5, 204 doi:10.1140/epjc/s10052-015-3397-6 [arXiv:1412.3989 [hep-ph]].

[2] H. Abramowicz et al. [H1 and ZEUS Collaborations], Eur. Phys. J. C 75 (2015) no.12, 580 doi:10.1140/epjc/s10052-015-3710-4 [arXiv:1506.06042 [hep-ex]].

[3] L. A. Harland-Lang, A. D. Martin, P. Motylinski and R. S. Thorne, Eur. Phys. J. C 76 (2016) no.4, 186 doi:10.1140/epjc/s10052-016-4020-1 [arXiv:1601.03413 [hep-ph]].

[4] R. S. Thorne, L. A. Harland-Lang and A. D. Martin, PoS DIS 2017 (2018) 202 doi:10.22323/1.297.0202 [arXiv:1708.00047 [hep-ph]].

[5] M. Aaboud et al. [ATLAS Collaboration], Eur. Phys. J. C 77 (2017) no.6, 367 doi:10.1140/epjc/s10052-017-4911-9 [arXiv:1612.03016 [hep-ex]].

[6] M. Goncharov et al. [NuTeV Collaboration], Phys. Rev. D 64 (2001) 112006 doi:10.1103/PhysRevD.64.112006 [hep-ex/0102049].

[7] E. L. Berger, J. Gao, C. S. Li, Z. L. Liu and H. X. Zhu, Phys. Rev. Lett. 116 (2016) no.21, 212002 doi:10.1103/PhysRevLett.116.212002 [arXiv:1601.05430 [hep-ph]].

[8] A. D. Martin, A. J. T. M. Mathijssen, W. J. Stirling, R. S. Thorne, B. J. A. Watt and G. Watt, Eur. Phys. J. C 73 (2013) no.2, 2318 doi:10.1140/epjc/s10052-013-2318-9 [arXiv:1211.1215 [hep-ph]].

[9] R. S. Towell et al. [NuSea Collaboration], Phys. Rev. D 64 (2001) 052002 doi:10.1103/PhysRevD.64.052002 [hep-ex/0103030].

[10] L. A. Harland-Lang, A. D. Martin and R. S. Thorne, Eur. Phys. J. C 78 (2018) no.3, 248 doi:10.1140/epjc/s10052-018-5710-7 [arXiv:1711.05757 [hep-ph]].

[11] G. Aad et al. [ATLAS Collaboration], JHEP 1502 (2015) 153 Erratum: [JHEP 1509 (2015) 141] doi:10.1007/JHEP02(2015)153, 10.1007/JHEP09(2015)141 [arXiv:1410.8857 [hep-ex]].

[12] J. Currie, E. W. N. Glover and J. Pires, Phys. Rev. Lett. 118 (2017) no.7, 072002 doi:10.1103/PhysRevLett.118.072002 [arXiv:1611.01460 [hep-ph]].

[13] M. Aaboud et al. [ATLAS Collaboration], JHEP 1709 (2017) 020 doi:10.1007/JHEP09(2017)020 [arXiv:1706.03192 [hep-ex]].

[14] G. Aad et al. [ATLAS Collaboration], Eur. Phys. J. C 76 (2016) no.10, 538 doi:10.1140/epjc/s10052-016-4366-4 [arXiv:1511.04716 [hep-ex]].

[15] [ATLAS Collaboration], ATL-PHYS-PUB-2018-017.

[16] M. Czakon, D. Heymes and A. Mitov, arXiv:1704.08551 [hep-ph].

[17] L. A. Harland-Lang, A. D. Martin, R. Nathvani and R. S. Thorne, arXiv:1907.02750 [hep-ph].

[18] A. Manohar, P. Nason, G. P. Salam and G. Zanderighi, Phys. Rev. Lett. 117 (2016) no.24, 242002 doi:10.1103/PhysRevLett.117.242002 [arXiv:1607.04266 [hep-ph]].

[19] A. V. Manohar, P. Nason, G. P. Salam and G. Zanderighi, JHEP 1712 (2017) 046 doi:10.1007/JHEP12(2017)046 [arXiv:1708.01256 [hep-ph]]. 